\title{
Adaptive Learning Environment System Based on Multi-event Driven Technology
}

\author{
https://doi.org/10.3991/ijet.v11i11.6250 \\ Jun-chang Zhang \\ Xuzhou Open University, Xuzhou, China
}

\begin{abstract}
Intelligent tutoring systems (ITSs) offer considerable flexibility in presentation of material and a greater ability to respond to idiosyncratic student needs. A multi agent system for adaptive learning environment is proposed in this paper. The system is composed of Open Agent Architecture and the Plan model. Teacher and student interaction was measured with the application of the system. The results prove that the system is an effective scheme to resolve the problem between dynamic students' needs of resources and static teaching resources.
\end{abstract}

Index Terms-distributed data, adaptive learning environment system, human tutor, multi-event driven technology

\section{INTRODUCTION}

With the rapid development of the Internet, more and more Internet business services have being deployed on the Web. This growing dependence upon Internet services underscores the importance of their availability and ability to handle large loads[1-2]. At the same time, exponential growth of the Internet population is placing unprecedented demands upon the scalability and robustness of these services. Staged Event-Driven Architecture (SEDA) [3-4] presents architecture for handling the massive concurrency and load conditioning demands of busy Internet services, which can support the extreme concurrency and burst applications. Therefore SEDA has become one of the hot fields in Web server performance optimization. In this paper, we review the progress and important achievements in the research of Web server performance optimization, and illustrate the significance of SEDA for promoting the development of Web techniques. We point out the main problems of SEDA in practical application. On the one hand, SEDA applies stages to execute the blocking operation, but the asynchronous API is insufficient to synchronized Java $\mathrm{I} / \mathrm{O}$, which can block all the stages easily and cause the bottleneck of system performance; on the other hand, SEDA, without a powerful global management role in its performance adjustment mechanism, cannot effectively coordinate the control policy between each stage.

The adaptive learning environment system based on a multi-event driven model adopts load-balance and feedback control as the core techniques. From the aspect of stage performance adjustment, heuristic optimization algorithm and initiative queue management algorithm are combined to optimize the stage management policies, including batch control for adjusting the number of events carried by working thread, service degradation to avoid the block between stages, self-feedback performance parameter adjustment thread resources of stages. From the aspect of global management, admittance control is ap- plied to avoid performance overload and load-balance clustering techniques are applied to coordinated thread resources and throughput performance between stage requests [5].

The two optimization processes can be effectively combined by feed-back mechanism. The self-adaptive mechanism is applied to the SEDA server architecture. By the way of improving the SEDA systematic control model and stage factor model resource distribution algorithm, the system control and stage adjustment are greatly enhanced. Based on the self-adaptive mechanism of the SEDA server architecture, the web server prototype system-OptiStage Server. Compared with other current common Web servers, on specific circumstances-high concurrency and high load, the OptiStage Server is more efficient on selfadapting and has high throughput [6].

Computers have been used in education for over 20 years. Computer-based training (CBT) and computer aided instruction (CAI) were the first such systems deployed as an attempt to teach using computers. In these kinds of systems, the instruction was not individualized to the learner's needs. Instead, the decisions about how to move a student through the material were script-like, such as "if question 21 is answered correctly, proceed to question 54; otherwise go to question 32." The learner's abilities were not taken into account. While both CBT and CAI may be somewhat effective in helping learners, they do not provide the same kind of individualized attention that a student would receive from a human tutor. For a computer based educational system to provide such attention, it must reason about the domain and the learner. This has prompted research in the field of intelligent tutoring systems (ITSs). ITSs offer considerable flexibility in presentation of material and a greater ability to respond to idiosyncratic student needs. These systems achieve their "intelligence" by representing pedagogical decisions about how to teach as well as information about the learner. This allows for greater versatility by altering the system's interactions with the student. Intelligent tutoring systems have been shown to be highly effective at increasing students' performance and motivation. For example, students using Smithtown, an ITS for economics, performed equally well as students taking a traditional economics course, but required half as much time covering the material.

\section{OVERVIEW}

For e-learning environments, "adaptive learning" or "learning path adaptation" is a critical issue to enhance teaching quality of an e-learning session. Adaptive learning provides "Subject Matter" content, adapted to a student's actual knowledge and learning style. Generally, elements of adaptive learning approaches include: moni- 
toring student activity, interpreting the results, understanding students' requirements and preferences, and using the newly gained information to facilitate the learning process.

So to build an adaptive learning environments needs the tutor able to supervise the activities of the learning entity (LE) with the possibility to take additional personalize exactions for the LE. Having a "Human Tutor" involved will ensure the needed "personal" contact for the LE in the e-learning environment. Tracing the student's step-by-step solution enables the tutor to provide personalized instruction in the problem solving context. Prototypically tutors provide immediate feedback on each problem solving action: recognizably correct actions are accepted and unrecognized actions are rejected. The proposed e-Tutor concepts do not try to diagnose student misconceptions and does automatically give problem solving advice. Instead, it allows the student maximum opportunity to reason about the current problem state, monitoring and assisting his/her approach. Generally, tutors do provide a feedback messages (hints) if the student appears confused about the nature of the current problem definition or a problem solving attempt. The proposed concept recognizes three general levels of advice: are minders of the current target, a general description of how to achieve the goal, and a description of exactly which problem solving action should be taken. Each of these three levels may be represented by multiple guidance steps [7-8].

Multi-agent methodology has recently appeared as an alternative to conceive Artificial Intelligence-based educational systems. The traditional architectures have proved to be too gigantic to deal with the new potential of systems that should be able to provide "learning anytime and anywhere" a web-based application that allows students to locate human experts and artificial resources available in the environment to get help during learning activities. This model is an example of a large-scale multi-agent learning environment several projects implement learning systems based on multi-agents architectures. Some of them work on a generic platform of agents.

Generally Java language used the web-based environment and occurred the CORBA platform and using agent. The multi-agent methodology can certainly bring several advantages to the development of educational applications since it deals well with applications where such crucial issues (distance, cooperation among different entities and integration of different components of software) are found. As a result, multi-agent systems (MAS), together with technologies of networking and telecommunications, bring powerful resources to develop educational systems. Aspects such as data persistence and mobility become extremely important in the design of this new class of educational systems. Besides, researchers in the educational field have shown that it is not possible to find a general strategy of teaching if we take into account human differences but it is rather probable to think that learning is an emergent result of rich and coherent interactions occurred during time.

For the computer simulation technology, its definition can started from the perspectives of evaluation and system performance, regards the enhancement of the overall performance as the base, and takes the computer model as the basis to carry out a comprehensive imitation and reference. Among these, regards the similarity of their inherent basic principles, techniques and specific computer applications as an opportunity. By computer technology, to improve

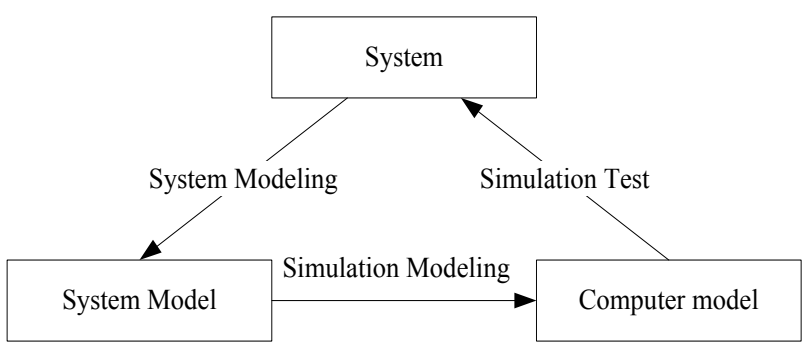

Figure 1. The basic framework

and repair its system performance comprehensively, then form a more comprehensive, multidisciplinary field computer application technology for the system.

However, in the computer simulation technology, the main elements of it can be divided into three parts. The computer simulation principle is shown as Fig. (1). First, it is the computer system, second is the construction of a comprehensive system model and the last is the application of computer technology. The specific implementation path is divided into three parts which are the establishment of the system model, construction of the simulation model and artificial process of the simulation experiment.

Model is the concrete abstraction of existing systems to create another kind of representations in their inner nature, thereby forming anti-corrupt cognitions and analysis of objective things. Through the multi-level conversion and integration process, the similarities between systems will be promoted and the desired end result will be produced.

Therefore SEDA has become one of the hot fields in Web server performance optimization. In this paper, we review the progress and important achievements in the research of Web server performance optimization, and illustrate the significance of SEDA for promoting the development of Web techniques. We point out the main problems of SEDA in practical application. On the one hand, SEDA applies stages to execute the blocking operation, but the asynchronous API is insufficient to synchronized Java $\mathrm{I} / \mathrm{O}$, which can block all the stages easily and cause the bottleneck of system performance. Material models are basically constructed through the similarities between systems - it means physical models can be built concretely. As for static material models, they are commonly built by general proportion models. For instance, architects often design and construct house models through reducing architectural proportions [9-11].

\section{METHOD AND ALGORITHM}

This section is about multi agent system for adaptive learning environment. Agent technology appears to be a promising approach to address the challenges of modern day educational environments, influenced enormously by advanced information and Internet technologies. It has seen a great recognition in quite a lot of educational and training computer-based activities. The existing world of education is currently changing rapidly in respect to all new technologies and methods coming up on the world. This change is taking place as well in technological as in instructional methods used in traditional and on-line education. Intelligent agents appeared to contribute rather important advantages for the scientific and educational computing. They have a major influence in different application fields of educational systems. They provide new educational paradigms, support theories, and happen to be 
rather helpful entities for both students and teachers in their computer-aided learning-teaching process. Their application in the educational field is mostly as personal assistants, user guides, alternative help systems, dynamic distributed system architectures, human-system mediators, and so forth. It consists of the students information, which students did choice the which lessons and levels and which lessons will be selected by the students in compulsory.

1) Adaptive Interaction Refers to adaptations that take place at the User interface level and are intended to facilitate or support the user's interaction with the learning platform, without, however, modifying in any way the learning "content" itself. OPUS One/OLAT allows to a certain extend to personalize the User Home page in enabling or disabling functions and setup the graphical appearance according to user needs.

2) Adaptive Course Delivery represents the most common used anthology of adaptation techniques applied in learning environments today. In particular, the idiom is used in reference to adaptations intended to alter a course (or, series of course sequences) to a specific learning entity. A major factor behind the implementation of adaptive techniques includes the compensation for the lack of a human tutor (who is capable of judging the learner capacity, goals, etc., and advising him on a personalized base), improving subjective evaluation of achievements by the learner. The most typical examples of adaptations in this category are: dynamic course (re-)structuring; adaptive navigation support; and, adaptive selection of alternative (sequences of) course material OPUS One e-Tutor facility and the integrated "Behavior Recorder" (BR) is supervising the learner in "real time" according to the learners" profile. If the learner encounters difficulties in solving course sequences (detected by the BR) or if the learner request tutoring, a "sequence (context specific)" of rule driven advise mechanism will be initiated and additional, tailored "problem solving" content will be proposed. This is considered an individual "exception" event and will be logged accordingly.

3) External Content Discovery refers to the discovery and storage of learning material / "content" from external sources, environments like other LMS's or specialized repositories. The adaptive component of this process lies with the integration and publication of this additional material among the course community. OPUS One / OLAT has the capability to detect, using the e-Tutor surveillance facility, the external search activity of the learning community, advising the learners to publish or share his additional material among his learning community and integrate the additional material in the course repository.

4) Adaptive Collaboration, refers to the involvement between multiple learners or group activities (and, therefore, social interaction), proposing collaboration towards common objectives. This is an important dimension to be considered as pedagogy is moving more and more towards collaborative approaches, which is what modern learning theory increasingly emphasizes, the importance of collaboration, cooperative learning, communities of learners and social negotiation Adaptive techniques can be used in this direction to facilitate the communication / collaboration process, ensure a good equivalent between learning communities. OPUS One / OLAT supports a variety of "tutored collaborative activities" integrated as components into a course or as standalone functionality. Functions like
Wiki, global or dedicated Forums, collaborative writing or collaborative assessment functions are just a few examples. In an exception case, the e-Tutor facility is able to propose additional collaborative actions to learners if the exception rules foresee such an action.

In random method, all nodes are randomly sorted and the node that sends request first will be scheduled first. The basic equation is shown as:

$$
\begin{aligned}
& \hat{f}_{H}^{\alpha}(x)=\frac{1}{\Gamma(1+\alpha)} \int_{-\infty}^{\infty} \frac{f(t)}{(t-x)^{\alpha}}(d t)^{\alpha} \\
& =\frac{1}{\Gamma(1+\alpha)} \int_{-\infty}^{\infty} f(t) g(x-t)(d t)^{\alpha} \\
& =f(x) * g(x),
\end{aligned}
$$

The equation is as follows:

$$
\partial_{j}\left(C_{i j k l} \partial_{k} u_{l}+e_{k i j} \partial_{k} \varphi\right)-\rho \ddot{u}_{i}=0
$$

Under the linear theory, that is:

$$
\partial_{j}\left(e_{i j k l} \partial_{k} u_{l}-\eta_{k i j} \partial_{k} \varphi\right)=0
$$

The linear equation can be expressed into the following simplified forms:

$$
\begin{gathered}
L(\nabla, \omega) f(x, \omega)=0, \\
L(\nabla, \omega)=T(\nabla)+\omega^{2} \rho \mathrm{J}(4)
\end{gathered}
$$

In which,

$$
\begin{array}{r}
T(\nabla)=\left\|\begin{array}{cc}
T_{i k}(\nabla) & t_{i}(\nabla) \\
t_{k}^{T}(\nabla) & -\tau(\nabla)
\end{array}\right\|, \mathrm{J}=\left\|\begin{array}{cc}
\delta_{i k} & 0 \\
0 & 0
\end{array}\right\|, \\
f(x, \omega)=\left\|\begin{array}{c}
u_{k}(x, \omega) \\
\varphi(x, \omega)
\end{array}\right\|(5)
\end{array}
$$

Consider delay, the L can be expressed as:

$$
L^{0}=\left\|\begin{array}{ll}
C_{i j k l}^{0} & e_{k i j}^{0} \\
e_{i k l}^{0 T} & -\eta_{i k}^{0}
\end{array}\right\|
$$

These functions can be expressed in the following form:

$$
\begin{gathered}
C(\mathrm{x})=C^{0}+C^{1}(\mathrm{x}), \quad e(\mathrm{x})=e^{0}+e^{1}(\mathrm{x}), \\
\eta(\mathrm{x})=\eta^{0}+\eta^{1}(\mathrm{x}), \quad \rho(\mathrm{x})=\rho_{0}+\rho_{1}(\mathrm{x})(7)
\end{gathered}
$$

The value with superscript of 1 represents the difference below:

$$
\begin{aligned}
& C^{1}=C-C^{0}, e^{1}=e-e^{0}, \\
& \eta^{1}=\eta-\eta^{0}, \rho_{1}=\rho-\rho_{0}
\end{aligned}
$$

The formula generates labels for each file block.

$$
\begin{gathered}
\operatorname{for}(j=0 ; j \leq n-1 ; j++) ; \\
\left\{W_{j}=r^{*}(j+1) ; T_{i}\right. \\
\left.=\left[h\left(W_{j}\right) * m_{j}\right]^{c} \bmod N\right\} ; \\
\text { Output }\left(T_{0}, T_{2}, \ldots T_{n-1}\right) ;
\end{gathered}
$$


And local fractional integral of $f(x)$ defined by Eq.12.

$$
\begin{aligned}
& { }_{a} I_{b}^{(\alpha)} f(t)=\frac{1}{\Gamma(1+\alpha)} \int_{a}^{b} f(t)(d t)^{\alpha} \\
& =\frac{1}{\Gamma(1+\alpha)} \lim _{\Delta t \rightarrow 0} \sum_{j=0}^{j=N-1} f\left(t_{\mathrm{j}}\right)\left(\Delta t_{\mathrm{j}}\right)^{\alpha}
\end{aligned}
$$

Our system is composed of the following modules, which interact using the Open Agent Architecture and the Plan model, a deliberative planning and execution monitoring module implemented using the Open Planning Architecture. The plan model is capable of performing onthe-fly plan repair when tutorial situations occur that have not been anticipated during the planning of the dialogue.

1) The NLU module, which translates the user's typed English input to a logical form. This is built using the CARMEL NLU toolkit, which includes a robust parser and a wide coverage grammar. We augmented CARMEL by adding a reference resolution module, and an interface to Word Net that can look for known synonyms of unknown words.

2) The generation module, which synthesizes English text from logical forms for each system turn. The generation module is a hybrid generator combining a templatebased approach with grammar-based processing within a pipeline of XML document transformations. The generation module built-in intelligence includes reasoning about implicit speech-acts, NP and VP pronominalize, and lexical choice.

\section{EXPERIMENT RESULT}

Some web-based learning systems, such as Blackboard, Moodle, SaKai, etc, provide the same learning resources to learners. According to user characteristics, personalized dynamic adaptive learning content don't presented. So the construction of adaptive learning system is an effective scheme to resolve the problem between dynamic students' needs of resources and static teaching resources. It is also an effective method to resolve the contradiction between overall infinite resources and individual needs of finite resources. It realizes two functions in the distance learning environment, which one is that learners can actively adapt distance learning, organize learning resources, make and carry out learning plan, select study strategy and can be self-assessment. Another is that system can provide learners with adaptive learning service support and in this learning circumstance, the system will put the students as service customers, as god and provide learning methods, strategies and emotional service. That is to say, adaptive learning system can recommend personal learning path and learning resources, according to students' characteristics and learning behaviors, such as learning styles, media types, learning interests, cognitive level, etc. It can effectively enhance students' individual education and improve the full development of a growing personality. Adaptive learning system is an ascendant research direction. It is possible that part of achievements has been widely used. However, the construction of system model is still very weak and this system also lacks the necessary empirical research.

The communicate model, a dialogue manager developed using the TRINDIKIT dialogue system shell. This module maintains the system's blackboard, including the dialogue history, and encodes rules of conversation (e.g., listeners are obliged to address questions). Note that knowledge of how to converse is kept separate from the planner's knowledge of how to tutor, and knowledge of the domain being tutored. BEER, a Basic Electricity and Electronics reasoned which encodes all of the system's knowledge about the subject matter to be tutored. BEER is able to compute answers to student questions and to simulate the execution of student lab actions.

The curriculum model involves a curriculum agent which encodes the representation of the teaching material. The material is annotated for learning goals, level of difficulty, and allocated time. BEESM, a situational modeler which infers relevant information about the immediate tutorial situation based on the student's interaction with the system (time elapsed, amount of material covered, student's answer correctness, etc.). BEESM derives values for the autonomy and approval to be given to the student, which inform the tutorial planning component that selects the next appropriate tutorial strategy, and the text generator BEETLEGEN that generates the appropriate linguistic realizations.

Each agent has a message-handling mechanism for incoming and out coming messages, to retrieve the data from an incoming message and to convert outgoing information to a common format. The agents exchange only the information attached to the message objects rather than the message objects. The messages are sent asynchronously without waiting for conformation of reception except where this is necessary, which is possible because the agents are implemented in different computers and their activities are parallel.

The communication protocol provides the possibility for sending a message to one only agent (point-to point), or to a group agents (multi-cast), or to all the agents in the system (broadcast). It is the sender who decides the type of outgoing message. Having a "Human Tutor" involved will ensure the needed "personal" contact for the LE in the e-learning environment. Tracing the student's step-by-step solution enables the tutor to provide personalized instruction in the problem solving context. Prototypically tutors provide immediate feedback on each problem solving action: recognizably correct actions are accepted and unrecognized actions are rejected. The proposed e-Tutor concepts do not try to diagnose student misconceptions and does automatically give problem solving advice. Instead, it allows the student maximum opportunity to reason about the current problem state, monitoring and assisting his/her approach. Generally, tutors do provide a feedback messages (hints) if the student appears confused about the nature of the current problem definition or a problem solving attempt.

Fig. 1 displays the underlying generic and modular architecture for the management tutorial dialogue. It is divided into four major parts (from left to right): external knowledge sources, the information state, the update engine and the three-layered planning and execution architecture. The architecture emphasizes the importance of clearly separating the knowledge sources involved in tutorial dialogue. The principle of the architecture proposed for an adapted training service is to allow the adaptation of knowledge transmission from a teaching function managing a virtual group of learners by a communication system (figure 2 and Table 1). 


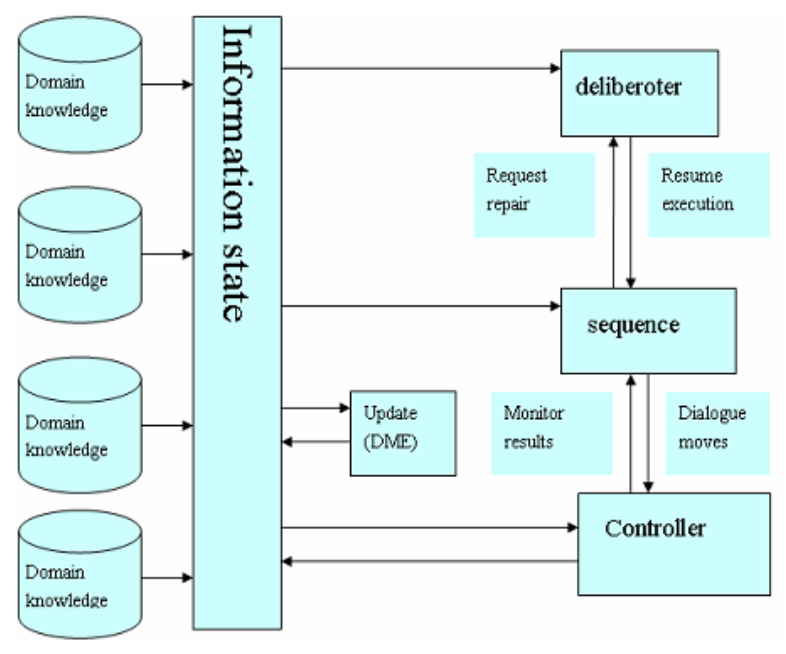

Figure 2. 3-level Architecture

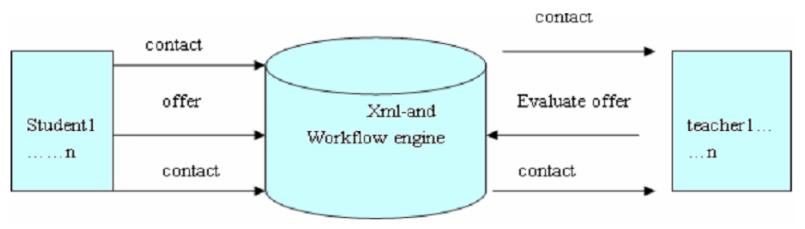

Figure 3. Teacher-student interaction in distance education

TABLE I.

TEACHER AND STUDENT INTERACTION DURING DistANCE EDUCATION ACTIVITIES

\begin{tabular}{|c|c|c|c|c|c|c|}
\hline \hline $\begin{array}{c}\text { University } \\
\text { types }\end{array}$ & $\mathbf{L 1 \%}$ & $\mathbf{L 2 \%}$ & $\mathbf{L 3 \%}$ & $\mathbf{L 7 \%}$ & $\mathbf{L 8 \%}$ & $\mathbf{L 9 \%}$ \\
\hline One & 53.3 & 0 & 0 & 0 & 0 & 0 \\
\hline Two & 0 & 5.3 & 0 & 6.7 & 0 & 0 \\
\hline Three & 33.3 & 23.7 & 0 & 0 & 0 & 0 \\
\hline Four & 0 & 47.7 & 7.15 & 3.3 & 0 & 0 \\
\hline Five & 6.7 & 2.6 & 0 & 43.3 & 0 & 0 \\
\hline Six & 6.7 & 21.1 & 46.4 & 6.7 & 14.3 & 0 \\
\hline Seven & 0 & 0 & 21.4 & 36.7 & 49.0 & 0 \\
\hline Eight & 0 & 0 & 17.9 & 3.3 & 30.6 & 34.8 \\
\hline Nine & 0 & 0 & 7.15 & 0 & 6.1 & 65.2 \\
\hline Total & 100 & 100 & 100 & 100 & 100 & 100 \\
\hline
\end{tabular}

\section{DISCUSSION}

Processing incoming messages requires two steps: (i) receiving, storing, and sorting messages; (ii) encoding message content for further processing by the agent in the context of a particular task. Processing an outgoing message similarly requires encoding of the information to be transmitted and actually mailing it in accordance with the exchange protocol.

Then, based-on object model and relation model in this paper has developed Adaptive Learning System by using JAVA, SQL Server and ontology technology, which is set as an example to introduce how learning objects and learning active sequence have been represented based-on user model, and introduce personal optimization recommendation of learning active sequence based-on the proposed algorithm, which students can avoid learning lost, reduce cognitive overload and the learning efficiency and quality can been improved. Based-on Adaptive Learning System, we analyze user behaviors objective variables and evaluate learning effectiveness from user-centered evaluations.

Our system can also be easily integrated with existing Web courseware or educational materials, thereby allowing reusability of existing courseware. The principle of the architecture proposed for an adapted training service is to allow the adaptation of knowledge transmission from a teaching function managing a virtual group of learners by a communication system (figure 2 and Table 1). Teaching functions are distributed by taking into account the participation of teachers, system, resources and learners in some cases (when a learner has the knowledge or experience necessary for playing the teacher's role). In the preliminary stage of the simulation program, the problem shall be defined scientifically. The clear mind and cautious psychology shall be maintained for its simulation analysis so as to clearly understand the specific problem that needs to be solved. In this process, the simulation problem is made by the simulation personnel, which requests the consignor to verify the described correctness. After this, its goals and corresponding specific plans shall be formulated scientifically. In this link, the corresponding practice for problem answering shall be reserved, thus to ensure the effective selection of the various strategies in the operation process.

The concept is able to support "human tutors" with accurate "learner centric" data to better qualify, judge and support the learner. The e-Tutor reliefs the "human tutor" from time consuming, low level tutoring interventions, supporting the learner directly with a variety of support tools. The "human tutor" can always overrule, add or modify e- Tutor proposed activities. Monitoring, support and tutoring capability of extensive collaborative functions (internal and external) allows a more fine grained adaptation/ personalization.

The generation module is a hybrid generator combining a template-based approach with grammar-based processing within a pipeline of XML document transformations. The generation module built-in intelligence includes reasoning about implicit speech-acts, NP and VP pronominalize, and lexical choice. If a system during its operating process has fixed certainty factors for accidents, this simulation process can be de-fined as deterministic simulation. Conversely, if accidents in a system's operating process do not own certainty factors but randomness, the process is usually defined as stochastic simulation. The result is fixed and unchanged during deterministic simulation while the result needs analyzing to form concrete similarity index in the latter process. As a commonly applied method of stochastic simulation, Monte Carlo simulation usually concretely constructs probabilistic model, then runs random detailed trials to solve mathematical problems. The results show that adaptive and personalized learning can improve learning effectiveness and students can complete the learning tasks using less time and learning objects. Meanwhile, students give a positive on learning enjoyment, learning effort, learning motivation and learning satisfaction in adaptive learning system. According to research progress and continual reflection, this thesis proposes that there are still some points which need to be studied in further. For example, we may study personalization recommendation among learners based-on collaborative filtering, which is helpful that students can find new knowledge and adaptive learning system may be applied to other subject teaching and basic education learners to evaluate learning effectiveness 
and how adaptive learning achievement may be migrated from E-learning to U-learning, which can be used effectively.

\section{CONCLUSION}

It has seen a great recognition in quite a lot of educational and training computer-based activities. The existing world of education is currently changing rapidly in respect to all new technologies and methods coming up on the world. In order to solve the problem of large delay and low energy efficiency in adaptive learning environment system, the author proposed a multi-event driven model. The material is annotated for learning goals, level of difficulty, and allocated time. BEESM, a situational modeler which infers relevant information about the immediate tutorial situation based on the student's interaction with the system (time elapsed, amount of material covered, student's answer correctness, etc.).

This change is taking place as well in technological as in instructional methods used in traditional and on-line education. Intelligent agents appeared to contribute rather important advantages for the scientific and educational computing. They have a major influence in different application fields of educational systems. By using this method, the "human tutor" can always overrule, add or modify e-Tutor proposed activities. Monitoring, support and tutoring capability of extensive collaborative functions (internal and external) allowed a more fine grained adaptation/ personalization. They provide new educational paradigms, support theories, and happen to be rather helpful entities for both students and teachers in their computer-aided learning-teaching process. Their application in the educational field is mostly as personal assistants, user guides, alternative help systems, dynamic distributed system architectures, human-system mediators, and so forth. Experiment results show that the proposed method can thus improve overall system performance substantially.

\section{REFERENCES}

[1] Y. Lin, J. Yang, Z. Lv, et al., “A self-assessment stereo capture model applicable to the internet of things," Sensors, vol. 15, no.8, pp. 20925-20944, August 2015. https://doi.org/10.3390/s15 0820925

[2] H. Jing, "The Study on the Impact of Data Storage from Accounting Information Processing Procedure," International Journal of Database Theory and Application, vol. 8, no.3, pp. 323-332, June 2015. https://doi.org/10.14257/ijdta.2015.8.3.28

[3] F. Chang, and C. Liu, "The study of optimal simulation and its application in vehicle scheduling for automatic space warehouse," Journal of System Simulation. vol.3, 1998.

[4] X. Dai, "A computer simulation tool for DEDS: A Case Study," Computer Simulation. vol. 4, 2001.

[5] W. Zhu, F. Qi, "Simulation and study status of complex logistics system," Journal of System Simulation. vol. 3, 2003.

[6] Y. Gandole, "Computer Modeling and Simulation of Ultrasonic System for Material Characterization," Modeling and Numerical Simulation of Material Science, Vol. 1 No. 1, pp. 1-13, 2011. https://doi.org/10.4236/mnsms.2011.11001

[7] J. Xu, L. Chen, and W. Gao et al., "CBTC Simulation Platform Design and Study". Journal of Computer and Communications, vol. 7, pp. 61-67, 2015. https://doi.org/10.4236/jcc.2015.39007

[8] J. Yang, M. Fan, G. Wang, et al., "Simulation Study Based on Somewhat Homomorphic Encryption," Journal of Computer and Communications, vol. 7, pp. 109-111, 2014. https://doi.org/10.4236/jcc.2014.22019

[9] Y. Wang, W. Zhu, H. Gen, et al., "Multi-Agent Based Social Integrity Simulation and Its Evolution," Sociology Mind, vol. 7, pp. 302-305, 2012. https://doi.org/10.4236/sm.2012.23040

[10] Y. Wang, A. Qi, and F. Cui, "Application of the Multimedia Teaching System Based on Real-time Shooting and Production in Martial Art Course," International Journal of Emerging Technologies in Learning, vol. 11, no.3, pp. 37-41, March 2016. https://doi.org/10.3991/ijet.v11i03.5347

\section{AUTHOR}

Jun-chang Zhang is with Xuzhou Open University, Xuzhou, China (43824451@qq.com).

Submitted 09 September 2016. Published as resubmitted by the author 15 October 2016. 